\title{
Renal Cell Carcinoma in Children: A Report of Three Cases
}

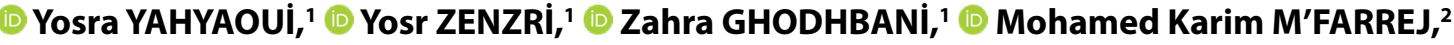

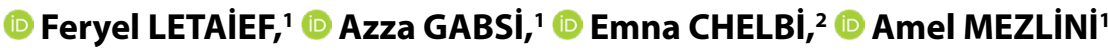

'Department of Medical Oncology, Salah Azaïz Institute, Tunis-Tunisia

${ }^{2}$ Department of Pathology, Mohamed Tahar Maamouri Hospital, Nabeul-Tunisia

\begin{abstract}
SUMMARY
Most renal cancers that occur in childhood and adolescence are nephroblastomas. Renal cell carcinoma is very rare in the pediatric population. It is highly aggressive and metastasizes rapidly. Consequently, the survival rates are poor. We report the cases of three adolescents aged 9, 14, and 18 years with renal cell carcinoma. We analyze through these observations the clinical, histological, and therapeutic characteristics of this entity.
\end{abstract}

Keywords: Adolescence; carcinoma, childhood; clear cell; renal cell.

Copyright $\odot$ 2021, Turkish Society for Radiation Oncology

\section{Introduction}

Renal cell carcinoma (RCC) is extremely rare in children. Literature on RCC in the pediatric and adolescent population is lacking. The incidence of this entity in childhood is estimated to be $0.1 \%-0.3 \%$ of all the neoplasm and $1.8 \%-6.3 \%$ of all renal cancers.[1] The therapeutic approach remains controversial. The cornerstone of the multidisciplinary treatment is surgery. Adjuvant treatment including chemotherapy, radiotherapy, and immunotherapy remains controversial. We report our experience of three cases with RCC.

\section{Case Report}

\section{Case 1}

An 18-year-old boy without any medical history presented with a flank pain and an abdominal mass. The body scan showed a $6-\mathrm{cm}$ right renal mass. He underwent radical nephrectomy with the complete resection of the primary tumor, and an abdominal CT scan was performed after the surgery. There were no metastases (Fig. 1).
The tumor size in the right kidney was $5.4 \times 4.4 \times 3$ $\mathrm{cm}$. It was well limited and associated to a satellite lymph node. On microscopic examination, a RCC with a solid, papillary, and glandular architecture was noted.

Tumor cells were large with clear-to-eosinophilic granular cytoplasm, atypical nuclei, and irregular nucleoli (Fig. 2). Psammoma bodies and highly vascularized tumor stroma were observed.

There were multiple and large necrosis areas without invasion of the perirenal fat, renal vein, hilum, or ureter. Tumor emboli and invasion of the pelvicalyceal system were noted.

The lymph node dissection found two metastatic paracaval lymph nodes: N2. The tumor was staged pT1bN2M0.

Immunohistochemical staining showed that the tumor cells were positive for CD10, EMA, Vimentin, and Cytokeratin. The diagnosis of renal cell carcinoma associated with Xp11.2 translocation/TFE3 gene fusion was confirmed (Figs. 3, 4).

Our patient did not receive adjuvant treatment. Cancer relapse occurred on the lungs and mediastinal lymph nodes one year after the surgery. 


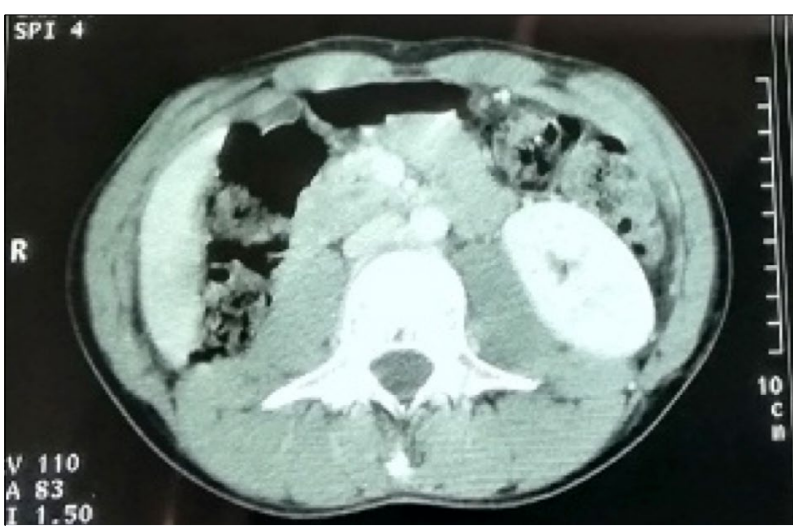

Fig. 1. Status post previous right nephrectomy.

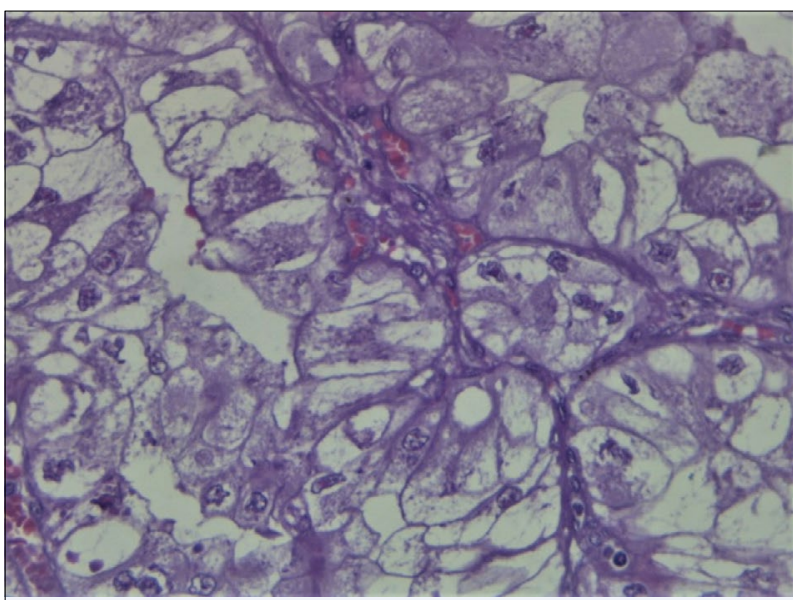

Fig. 2. Polyhedral tumor cells with prominent plasma membranes and clear or eosinophilic and finely granular cytoplasm. Nuclei are irregular with clearly visible nucleoli (Hematoxylin Eosin 400×).

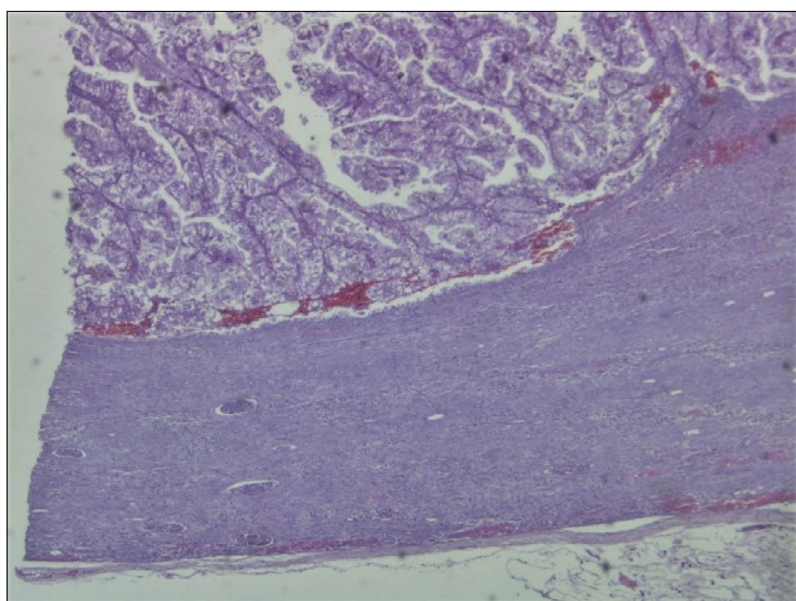

Fig. 3. Transcription factor E3 (TFE3) translocation renal carcinoma papillary architecture with thin walled blood vessels and clear cells (Hematoxylin Eosin 40x).

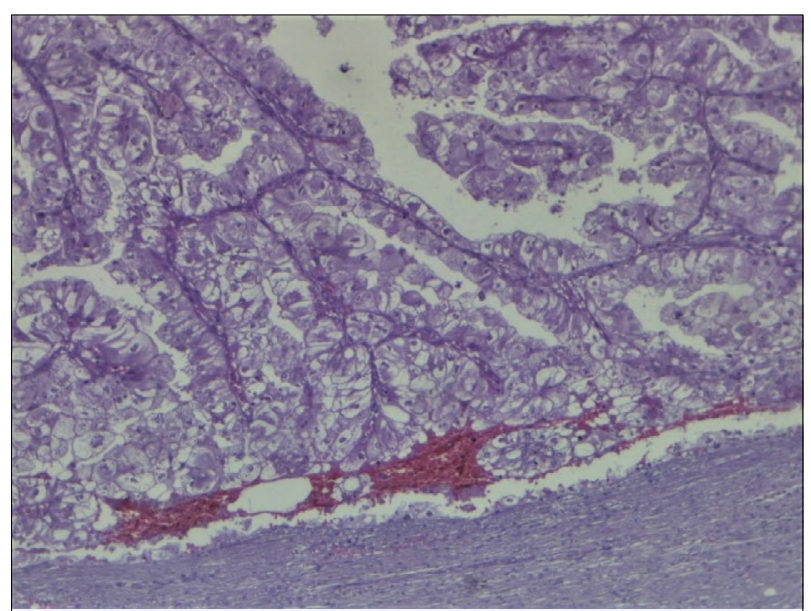

Fig. 4. Transcription factor E3 (TFE3) translocation renal carcinoma papillary architecture with thinwalled blood vessels and clear cells (Hematoxylin Eosin 100×)

Our patient received oral Sunitinib once daily (50 mg/day) for four weeks every six weeks. CT scan showed progression of the disease. He received Vinblastine as a second line treatment with adrenal, bone, and lung progression. A palliative chemotherapy (Gemcitabine+Doxorubicine) associated with zoledronic acid was administered with disease progression. Our patient died after the third line treatment.

\section{Case 2}

A 14-year-old boy without comorbidities presented with an isolated right thigh pain, noticed several days before his admission to the hospital. MRI of the thigh showed a rapidly evolving lesion of the femoral proximal diaphysis without soft tissue invasion. An abdominal computed tomography (CT) showed a left mediorenal mass with a maximum diameter of $30 \mathrm{~mm}$. The bone scintigraphy showed metastases in the L5 vertebra, right femur, left sacrum, and ilium. A bone biopsy was performed. Histological examination revealed a RCC metastasis. Large polygonal cells with round-toelongated nuclei, abundant cytoplasm, and multiple areas of necrosis were observed. Immunohistochemistry was performed using monoclonal antibodies against EMA, Cytokeratine, Actine, HMB45, CD99, PS100, and Chromogranin A. Immunohistochemical analysis of our patient depicted large cells positive for EMA. The patient underwent transabdominal radical nephrectomy with complete resection of the primary tumor.

The tumor size in the left kidney was $25 \times 20 \mathrm{~mm}$. Spindle cells arranged in fascicles were observed. Large cells with eosinophilic cytoplasm, ovoid nuclei, and 
large and hyperchromatic nucleolus were noted. The renal vein, hilum, and ureter were free of any invasion.

The tumor was a grade 4 pT1a with a fusocellular sarcomatoid component. Immunohistochemical staining showed that the tumor cells were positive for CD10 and Vimentin. Stains for CD117 and CK7 were negative.

The genetic study of the tumor cells revealed a deletion of the VHL gene without TFE3 and TFEB rearrangements. The histopathological, immunohistochemical, and cytogenetic profile of this neoplasm confirmed the diagnosis of clear-cell carcinoma T1aNXM2: stage IV.

The treatment involved a radical surgery followed by a tyrosine kinase inhibitor: Sunitinib once daily $(50$ $\mathrm{mg}$ /day) orally for four weeks every six weeks associated with monthly zoledronic acid. A progression of the disease was noted after six months of treatment. Lung metastases were noted in the chest CT scan. He underwent a second line treatment with Interferon and Vinblastine, and the CT scan still showed progression of the disease. The patient died before starting the third line treatment.

\section{Case 3}

A 9-year-old girl without any medical history presented to the emergency department with a 3-month history of macroscopic hematuria and flank pain. Ultrasound showed a renal mass in the lower pole of the right kidney with a diameter of $68 \mathrm{~mm}$ (Fig. 5). An abdominal computed tomography (CT) showed a tumor originating from the lower pole of the right kidney with a maximal diameter of $69 \mathrm{~mm}$ (Figs. 6, 7). No distant metastases were detected. Renal biopsy revealed a clear-cell carcinoma Fuhrman Grade 1. She underwent a radical nephrectomy with complete resection of the primary tumor. The tumor size was $70 \times 60 \times 50$ $\mathrm{mm}$. Polygonal tumor cells with clear or eosinophilic cytoplasm were observed. The nuclei of the tumor cells were round, large, and irregular with nucleoli visible at $\times 100$. The stoma contains a mixed inflammatory infiltrate consisting of lymphocytes and plasma cells. The sarcomatoid component accounted for $30 \%$ of the tumor volume. Tumor necrosis extended beyond the renal capsule and invaded the fat tissue. No tumor emboli or renal vein invasion were observed. The histopathological examination of this neoplasm confirmed the diagnosis of clear-cell carcinoma with sarcomatoid component pT3aN0. The patient is undergoing adjuvant chemotherapy. The multidisciplinary decision was to treat her based on high-risk regimens used in Wilms tumor including the following drugs: Cyclophosphamide, Carboplatin, Etoposide, and Doxorubicin.

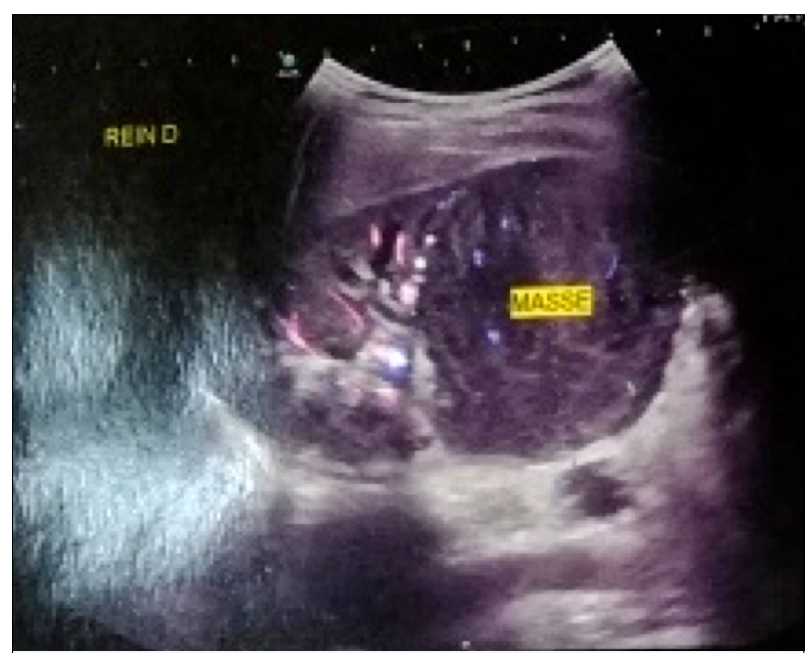

Fig. 5. Renal mass in the lower pole of the right kidney.

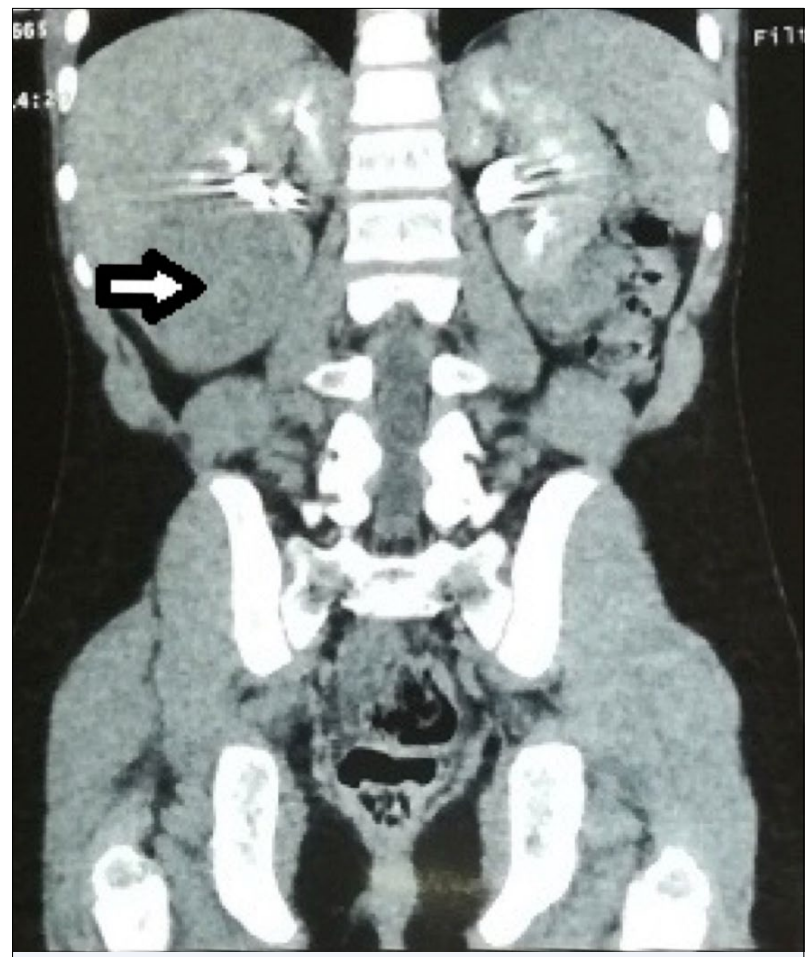

Fig. 6. Computed tomography (CT) shows a tumor originating from the lower pole of the right kidney.

\section{Discussion}

Renal cell carcinoma (RCC) is uncommon in childhood, and its incidence increases with age. The main differential diagnosis is Wilms tumor (nephroblastoma). However, an age above 10 years at admission must alert the clinician to the risk of RCC.

RCC accounted respectively for $1.4 \%, 15.2 \%$, and $52.6 \%$ of cancers in children younger than 4 years, 


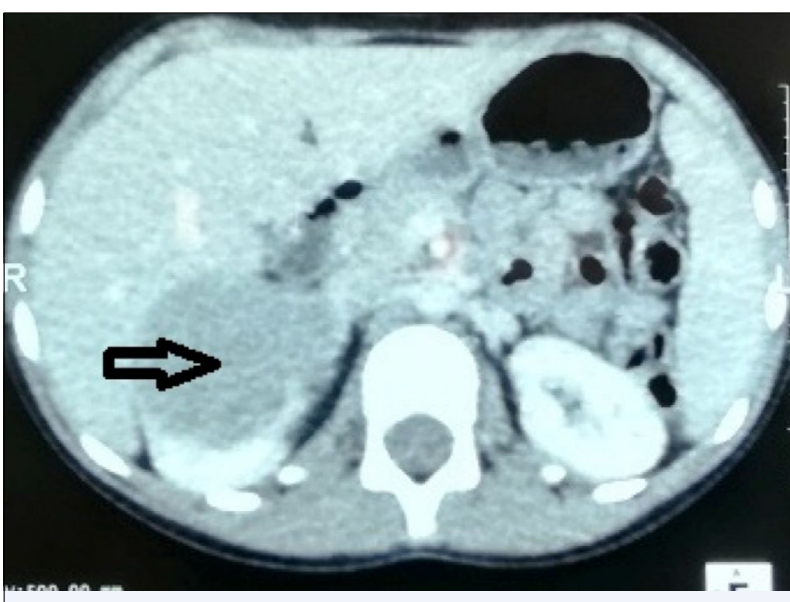

Fig. 7. Computed tomography (CT) shows a tumor originating from the lower pole of the right kidney.

aged 5 to 9 years, and 10 to 15 years according to a survey of the Japanese Society of Pediatric Surgeons. [2] There is no sex predominance for this entity during childhood; however, a male predominance is reported in adults.[3]

The clinical aspects of RCC in pediatrics patients are the same as those in adults, including flank pain, abdominal mass, hematuria, fever, and weight loss.[4]

Tuberous sclerosis, urogenital malformations, chronic renal failure, neuroblastoma, and teratoma with chemotherapy may be associated with RCC.[5] RCC is characterized by a localized tumor (69\%) and regional lymph node metastases (16\%) or distant metastases (8\%) according to the previously published litרerature.[6] Rarely, RCC is revealed by metastasis as in our patient aged 14 years. The most frequent metastatic sites are lungs (40\%-65\%), liver $(35 \%-57 \%)$, and bones $(10 \%-42 \%)$. [7]

RCC are classified as clear-cell, papillary, chromophobe, Xp 11-translocation RCC, RCC associated with neuroblastoma, and unclassified RCC according to WHO classification (2004 classification).

The incidence of the pathological subtypes of RCC include papillary $(33 \%)$, translocation type $(22 \%)$, unclassified (16\%), and rarely clear-cell.[8] The second and third patients had the very rare clear-cell histological subtype. Clear-cell RCC is frequent in patients with Von Hippel-Lindau disease and is characterized by its relatively hypervascular nature.[9] But, clear-cell RCC can be seen in childhood even without VHL.[10]

Xp11.2 translocation renal cell carcinomas are uncommon tumors. They usually affect children and adolescents. They result from gene fusions between the transcription factor E3 (TFE3) gene located and at least
6 various partners. A Meta-analysis found that RCC TFE3 positive patients had a poor prognosis (overall survival and disease-free survival) and a higher stage (III/IV) than RCC TFE3 negative patients. Xp11.2 translocation carcinomas appear to have a poorer prognosis than non-Xp11.2 translocation carcinomas in young adults and children. Therefore, those patients must benefit from a much aggressive treatment.[11] The first patient had Xp11.2 translocation and an aggressive clinical course.

The cornerstone of the multidisciplinary treatment of localized RCC is surgery. The role of surgery is decisive in the success of the treatment. The resection should be complete. Survival rate is significantly increased when margins are negative.[1] The role of preoperative or postoperative radiotherapy is uncertain.

RCC is resistant to radiation therapy or chemotherapy. The treatment of the metastases remains unclear. Angiogenesis-inhibiting or immune-stimulating agents, like vaccines, Interferon, Sorafenib, or Sunitinib are possible therapeutic alternatives, with limited results because of the lack of any prospective randomized studies. Cytokines such as IL- 2 and IFN- $\alpha$ provide modest overall survival benefits.[12]

The prognosis in advanced stages of RCC is poor. All patients with stage IV disease died in a series report by Lack et al.[13]

\section{Conclusion}

Renal cell carcinoma is extremely rare in children. The multidisciplinary approach is mandatory. Clinical history and pathological examination are necessary to establish a conclusive diagnosis. The treatment of choice for localized RCC is radical nephrectomy. The prognosis in advanced stages of RCC is poor.

Informed consent: Written informed consent was obtained from the patient for the publication of the case report and the accompanying images.

Peer-review: Externally peer-reviewed.

Conflict of Interest: The authors declare no conflict of interest.

Authorship contributions: Concept - Y.Y., Y.Z., Z.G., E.C., A.M., ; Design - Y.Z., Z.G., M.K.M.; Supervision - Y.Y., A.M.; Data collection \&/or processing - Y.Z., Z.G.; Analysis and/or interpretation - Y.Y., Y.Z., Z.G., ; Literature search Y.Y., Y.Z., Z.G.; Writing - Y.Y., Y.Z., Z.G..; Critical review F.L., A.G. 


\section{References}

1. Louati H, Jlidi S, Charieg A, Hannechi W, Ahmed YB, Nouria F. Renal Cell Carcinoma in Children: About Two Case Report. Med Sur Urol 2016;5:4.

2. Uchiyama $M$, Iwafuchi $M$, Yagi $M$, Iinuma $Y$, Ohtaki M, Tomita Y, et al. Treatment of childhood renal cell carcinoma with lymph node metastasis: two cases and a review of literature. Journal of surgical oncology 2000;75(4):266-9.

3. Abdellah A, Selma K, Elamin M, Asmae T, Lamia R, Abderrahmane $\mathrm{M}$, et al. Renal cell carcinoma in children: case report and literature review. Pan Afr Med J 2015;20:84.

4. Dénes FT, Duarte RJ, Cristófani LM, Lopes RI. Pediatric genitourinary oncology. Front Pediatr 2013;1:48.

5. Chauhan NS, Bharti R, Chander B, Kumar S. Pediatric Clear Cell Renal Cell Carcinoma with Pelvicalyceal System Invasion and Contiguous Extension Upto Bladder: Novel and Bizarre Tumor Behaviour. Polish journal of radiology 2016;81:256-60.

6. Chauhan NS, Bharti R, Chander B, Kumar S. Pediatric Clear Cell Renal Cell Carcinoma with Pelvicalyceal System Invasion and Contiguous Extension Upto Bladder: Novel and Bizarre Tumor Behaviour. Polish journal of radiology. 2016;81:256-60.

7. Carcao MD, Taylor GP, Greenberg ML, Bernstein ML, Champagne M, Hershon L, et al. Renal-cell carcinoma in children: A different disorder from its adult counterpart? Medical and Pediatric Oncology 1998;31(3):153-8.

8. Selle B, Furtwängler R, Graf N, Kaatsch P, Bruder E, Leuschner I. Population-based study of renal cell carcinoma in children in Germany, 1980-2005: more frequently localized tumors and underlying disorders compared with adult counterparts. Cancer 2006;107(12):2906-14.

9. Chung EM, Lattin GE Jr, Fagen KE, Kim AM, Pavio MA, Fehringer AJ, et al. Renal Tumors of Childhood: Radiologic-Pathologic Correlation Part 2. The 2nd Decade: From the Radiologic Pathology Archives. Radiographics 2017;37(5):1538-58.

10. Bruder E, Passera O, Harms D, Leuschner I, Ladanyi $\mathrm{M}$, Argani P, et al. Morphologic and molecular characterization of renal cell carcinoma in children and young adults. The American journal of surgical pathology 2004;28(9):1117-32.

11. Rao Q, Guan B, Zhou X-j. Xp11.2 Translocation Renal Cell Carcinomas Have a Poorer Prognosis Than Non-Xp11.2 Translocation Carcinomas in Children and Young Adults: A Meta-analysis. Int J Surg Pathol 2010;18:458-64.

12. Ambalavanan M, Geller JI. Treatment of advanced pediatric renal cell carcinoma. Pediatric Blood \& Cancer 2019;66(8):e27766.

13. Lack EE, Cassady JR, Sallan SE. Renal cell carcinoma in childhood and adolescence: a clinical and pathological study of 17 cases. J Urol. 1985;133(5):822-8. 